\title{
OLIMPISMO Y EDUCACIÓN PARA LA PAZ: UN ANÁLISIS HISTÓRICO ${ }^{1}$
}

\author{
Christina Koulouri \\ Profesor de Historia Moderna y Contemporánea \\ Jefe de Departamento de Ciencias Políticas e Historia, \\ Universidad Panteion de Ciencias Sociales y Políticas \\ Atenas (Grecia) \\ christina.koulouri@panteion.gr \\ Fecha de recepción: junio 2019 \\ Fecha de aceptación: septiembre 2019
}

DOI: $\underline{\text { http://doi.org/10.15366/citius2019.12.2.003 }}$

\section{Resumen:}

En este documento se ha intentado investigar los vínculos históricos entre el olimpismo y la búsqueda de la paz, tanto exitosa como sin éxito, y las formas en que la educación olímpica, como parte integrante del olimpismo, incorporó la educación para la paz.

A finales del siglo XIX apareció un fuerte movimiento pacifista con la fundación de sociedades pacifistas nacionales en Europa occidental y central y la convocatoria de conferencias anuales por parte de los consejos nacionales de paz. La organización del Congreso Internacional de Paz en París y el establecimiento de la Oficina Internacional de Paz en 1889 fueron un punto de inflexión en el movimiento por la paz. Los principios primordiales del olimpismo y la estructura organizativa de los Juegos Olímpicos modernos pueden entenderse mediante la comparación con los esfuerzos internacionalistas contemporáneos, principalmente el «internacionalismo idealista». La estructura organizativa del movimiento olímpico se basó en el modelo de otros programas internacionalistas. El movimiento olímpico ha incorporado en sus valores el papel que debe desempeñar la educación a través del deporte y concede importancia a la función educativa del deporte. Los valores olímpicos evolucionaron en el siglo XX, para corresponder al modelo de ciudadano de una democracia moderna, sentando las bases de la educación olímpica. En una sociedad globalizada, donde la competencia por la distinción a cualquier costo se está haciendo más fuerte, la educación, no sólo la educación olímpica, debe desarrollar un espíritu de colaboración y espíritu de equipo en los niños desde una edad temprana. Los educadores pueden ser aquellos que están llamados principalmente a actuar como «embajadores de la paz», ya que se comprometen a preparar a las próximas generaciones para vivir en armonía, planeando un futuro donde la paz sea más probable que la guerra, en lugar de una sociedad utópica.

Palabras Clave: Paz, Educación para la paz, Olimpismo, Movimiento Olímpico.

\footnotetext{
${ }^{1}$ El presente trabajo es la versión en español de la conferencia que fue presentada en inglés por la autora en la 15th Session for Presidents or Directors of National Olympic Academies organizada por la Academia Olímpica Internacional, en su sede de Olimpia en mayo del 2019.
} 


\title{
Title: OLYMPISM AND PEACE EDUCATION: A HISTORICAL ANALYSIS
}

\begin{abstract}
:
In this paper I shall attempt to investigate the historical links between Olympism and the pursuit of peace, both successful and not, and the manners in which Olympic education, as an integral part of Olympism, incorporated peace education.

A strong pacifist movement appeared at the end of the $19^{\text {th }}$ century with the founding of national pacifist societies in Western and central Europe and the convening of annual conferences by national peace councils. The organization of the first International Peace Congress in Paris and the establishment of the International Peace Bureau in 1889 were a turning point in the peace movement. The main principles of Olympism and the organizational structure of the Modern Olympic Games can be understood through the comparison with contemporary internationalist endeavours, mainly "idealistic internationalism". The organizational structure of the Olympic Movement was based on the model of other internationalist programmes. The Olympic Movement has incorporated these values as regards the role that education must play through sports and attaches importance to the educational function of sports. Olympic values evolved in the $20^{\text {th }}$ century, so as to correspond to the model of a citizen of a modern democracy, laying the foundations of Olympic education. In a globalized society, where competition for distinction at any cost is becoming stronger, education - not solely Olympic education - must develop a spirit of collaboration and team spirit in children from a young age. Educators can be those that are chiefly called upon to act as "ambassadors of peace", as they undertake to prepare the next generations to live in harmony, planning a future where peace is more likely than war, rather than a Utopian society.
\end{abstract}

Key Words: Peace, Education for peace, Olympism, Olympic Movement.

\section{Introducción}

En 1889, en uno de sus primeros discursos, Coubertin describió la educación física como un sistema educativo con alcance, método y reglas específicos. La dimensión moral de este sistema era especialmente importante porque los deportes condujeron, en su opinión, a una victoria de la voluntad y a cumplir el ideal humano. Poco a poco, y sobre la base de los mismos principios, ${ }^{2}$ Coubertin concibió el olimpismo principalmente como una reforma del sistema de educación, cuyo objetivo era fomentar un conjunto de valores morales entre los jóvenes a través de concursos deportivos y los deportes. Las dimensiones educativas y morales del deporte estaban vinculadas, en la mente del fundador del olimpismo, a la búsqueda de la comprensión global y la paz. El pacifismo imbuyó la postura moral que el olimpismo abrazó, tanto a nivel individual como para la humanidad. En sus numerosos textos, Coubertin subrayó el papel que los Juegos Olímpicos pueden desempeñar en la preservación de la paz entre los pueblos. Desde entonces, la paz ha constituido uno de los valores olímpicos y se incorpora a todos los textos y programas del olimpismo, incluida la Carta Olímpica, en sus diversas versiones y en los discursos de los principales representantes del Movimiento Olímpico. Sin embargo, en el siglo XX las políticas pacifistas se enfrentaron a la realidad de dos guerras mundiales y muchos conflictos regionales, la bipolaridad de la guerra fría y los sucesivos boicots olímpicos. Este documento recoge mis intentos por investigar los vínculos históricos entre el olimpismo y la búsqueda de la paz, tanto exitosa como sin éxito, y las formas en que la educación olímpica, como parte integrante del olimpismo, ha incorporado a la educación para la paz.

\footnotetext{
${ }^{2}$ G. Rioux (Ed.), Pierre de Coubertin. Textes choisis, vol. 1: Révélation, Weidmann, Zurich- Hildersheim-New York, pp. 13-14.

Citius, Altius, Fortius Volumen 12 ne 2. Noviembre, 2019. ISऽN. 2340-9886.

http://doi.org/10.15366/eitius2019.12.2
} 


\section{Olimpismo y paz mundial}

A la temprana edad de 21 años, Coubertin se integró en las organizaciones francesas por la Paz Social (en la Union de la paix sociale), fundada en 1872 por Frédéric Le Play y que estaba muy de moda entre los círculos conservadores liberales. El pensamiento de Le Play, que restó importancia a la política y al papel del Estado en la consecución de la reforma social, afectó claramente a Coubertin, en cuanto a su falta de confianza hacia la política y le dio lugar a dar forma a una doctrina, el Olimpismo, que proclamó neutralidad política. ${ }^{3}$ Unos años más tarde, cuando se organizó el Congreso por la Paz en París, Coubertin se familiarizó con las actividades del movimiento pacifista y trabajó en un programa de reforma destinado a la paz mundial a través de la educación de la futura élite. ${ }^{4}$ Por lo tanto, el olimpismo nació a través de una relación directa con los movimientos pacifistas europeos, que estaban en su apogeo en esa época, hasta el estallido de la Primera Guerra Mundial. ${ }^{5}$

De hecho, a finales del siglo XIX apareció un fuerte movimiento pacifista con la fundación de sociedades pacifistas nacionales en Europa occidental y central y la convocatoria de conferencias anuales por parte de los consejos nacionales de paz, así como un mensaje de paz que se transmitió en las escuelas principalmente a través de iniciativas de activistas franceses. La organización del Congreso Internacional de Paz en París y el establecimiento de la Oficina Internacional de Paz en 1889 fueron un punto de inflexión en el movimiento por la paz. Alfred Nobel, en 1896, dejó dinero en su testamento a aquellos que «habrán hecho la mayor o mejor obra para la fraternidad entre las naciones, para la abolición o reducción de los ejércitos permanentes y para la celebración y promoción de congresos de paz», como el Palacio de la Paz en La Haya y el Edificio de la Unión Panamericana en Washington. ${ }^{6}$

Por lo tanto, los principios esenciales del olimpismo y la estructura organizativa de los Juegos Olímpicos modernos pueden entenderse mediante la comparación con los esfuerzos internacionalistas contemporáneos, principalmente el «internacionalismo idealista», que comprendía la reforma internacional con dimensiones políticas, sociales y culturales. El propósito de estos internacionalismos era la colaboración internacional y la paz a través de la reunión y la asociación mutua de diferentes pueblos. En contraste con el internacionalismo socialista, los «internacionalismos idealistas» promovieron la neutralidad política como su característica clave, mientras que no rechazaron el amor a la patria. En 1901, Coubertin expresó claramente el tipo de internacionalismo que él defendía: ${ }^{7}$

«Hay dos formas de entender el internacionalismo. Uno es el de los socialistas, los revolucionarios, y en general de los teóricos y utópicos [...] La segunda es la de los hombres, que hacen observaciones sin prejuicios y tienen en cuenta la realidad, más que sus ideas favoritas. Desde hace mucho tiempo, estos hombres se han dado cuenta de que las características nacionales son una condición indispensable para la vida de un pueblo y que, lejos de debilitarlos... el contacto con otras personas los hace más agudos» ${ }^{8}$.

\footnotetext{
${ }^{3}$ P. Clastres - P. Dietschy - S.Laget, La France et l'Olympisme, Paris : ADPF, 2004, pp.32-34. In 1931, in his text, Coubertin recognized what he owed to these two men, Le Play and Arnold.

${ }^{4}$ Ibid., $47-49$.

${ }^{5}$ Sandi E. Cooper, Patriotic Pacifism. Waging War on War in Europe 1815-1914, New York-Oxford: Oxford University Press, 1991, p. 89.

${ }^{6}$ Ibid., Pp. $79-87$

${ }^{7}$ Ver John Hoberman, «Toward a Theory of Olympic Internationalism», Journal of Sport History 22/1 (spring 1995 ), p. 5.

8 «Notes sur l'éducation publique» (1901), 262-263 in N. Müller (Ed.) Pierre de Coubertin 1863-1937 - Olympism: Selected Writings. Lausanne: International Olympic Committee, 2000, p. 45, note 41.
} 
El equilibrio entre internacionalismo y patriotismo que se encuentra en los escritos de Coubertin refleja los principios clave del movimiento pacifista de esa época, que reconoció la diversidad de las naciones y el conflicto como parte de la acción humana; pero planteó la necesidad de una solución «civilizada» en lugar de guerra. Este fue el «pacifismo patriótico» que predominó en el movimiento pacifista internacional antes de la Primera Guerra Mundial. ${ }^{9}$ El renacimiento de los Juegos Olímpicos formó parte de un plan pacifista, que combinaba el internacionalismo con el patriotismo, como lo describió Coubertin en el Congreso de París de 1894, donde también declaró, entre otras cosas:

«Con la restauración de los Juegos Olímpicos, sobre cimientos y bajo condiciones de acuerdo con las necesidades de la vida moderna, reuniríamos a representantes de las naciones del mundo cada cuatro años. Cabe esperar que estos pacíficos enfrentamientos corteses son la mejor forma de internacionalismo» ${ }^{10}$.

La afiliación ideológica del olimpismo con el movimiento pacifista internacional también se puede ver en los participantes del Congreso de la Sorbona. Aproximadamente una cuarta parte de los cincuenta miembros honorarios del Congreso tenía algunos vínculos con la Oficina Internacional de Paz (1891) y con la Mesa de la Unión Interparlamentaria (1892). ${ }^{11}$ De hecho, en 1892 en Roma, en la Conferencia por la Paz, el pacifista inglés Hodgson Pratt formuló la idea de «concursos deportivos» entre estudiantes de todas las universidades de todo el mundo como factor de fraternidad, mientras que Frédéric Passy, más tarde el primer ganador del Premio Nobel de la Paz, había señalado tales competiciones deportivas como «reuniones pacíficas de las naciones» (le rendez-vous pacifique des nations). ${ }^{12}$

La estructura organizativa del Movimiento Olímpico se basó en el modelo de otros programas internacionalistas: designación de un órgano central -el Comité Olímpico Internacional-, en el que representantes de varios países participan en igualdad de condiciones y que se relaciona con organismos similares correspondientes en cada país; la organización periódica de conferencias internacionales en el marco de la iniciativa y bajo la supervisión del órgano central; la definición y codificación de las normas y los términos para participar en competiciones deportivas. ${ }^{13}$

Mientras el Olimpismo fue evolucionando, fue estableciendo sistemáticamente un sistema de valores e inventó un lenguaje de símbolos y rituales que fueron dando sustancia física al contenido pacifista abstracto de su ideología y lo hacen accesible a quienes participan en el evento de los Juegos Olímpicos únicamente como espectadores. ${ }^{14}$ Tales símbolos incluyen los anillos olímpicos y la bandera olímpica, el himno olímpico y el juramento olímpico, mientras que rituales, como el relevo de la antorcha, las ceremonias de apertura y clausura de los Juegos, y el desfile de atletas incorporados en ellos, juegan un papel similar. ${ }^{15}$ Los anillos olímpicos, que también están representados en la

\footnotetext{
${ }_{9}^{9}$ Dietrich R. Quanz, «Civic Pacifism and Sports-Based Internationalism: Framework for the Founding of the International Olympic Committee», Olympika: The International Journal of Olympic Studies 2 (1993), p. 9-10; Cooper, Patriotic Pacifism, op.cit.

${ }^{10}$ N. Müller (ed.), Pierre de Coubertin 1863-1937, op.cit., pp. 298-299.

${ }^{11}$ Quanz, «Civic Pacifism...», op.cit.

${ }^{12}$ P. Clastres - P. Dietschy - S. Laget, Op.cit., P. 54.

${ }^{13}$ Christina Koulouri, «Introduction: Rewriting the History of the Olympic Games» in idem (ed.), Athens, Olympic City, 1896-1906, Athens: International Olympic Academy, 2004, pp. 36-40.

${ }^{14}$ Ver John MacAloon, «Olympic Ceremonies as a Setting for Intercultural Exchange» in M. De Moragas -J. MacAloon M. Llinés (eds.), Olympic Ceremonies. Historical Continuity and Cultural Exchange, Lausanne: IOC, 1996, p. 32.

${ }^{15}$ Sobre el establecimiento de los diferentes símbolos olímpicos y la organización correspondiente de las ceremonias olímpicas, ver De Moragas -MacAloon - Llinés (eds.), Olympic Ceremonies, op.cit., Principalmente pp. 114-118.
} 
bandera olímpica, fueron diseñados por el propio Coubertin en 1913 y fueron presentados en el VI Congreso Olímpico Internacional en París en 1914, que celebró el vigésimo aniversario del Movimiento Olímpico. Los cinco anillos, en cinco colores diferentes, simbolizan «las cinco partes del mundo» y junto con el blanco de la bandera, todas las naciones sin excepción. Por lo tanto, es un «emblema verdaderamente internacional»: todas las naciones y todos los continentes están representados sin distinciones en la institución internacional de los Juegos Olímpicos. ${ }^{16}$ Las palomas lanzadas durante la ceremonia de clausura de los primeros Juegos Olímpicos de Atenas y posteriormente en las ceremonias de apertura de 1920 también aludieron a la paz. ${ }^{17}$

Después del final de la Primera Guerra Mundial, la presión por la paz internacional fue grande y las consecuencias de la guerra en el Movimiento Olímpico fueron visibles. Los Juegos Olímpicos de Berlín programados para 1916 fueron cancelados. Alemania fue excluida de los Juegos Olímpicos de Amberes 1920, así como de los Juegos Olímpicos de 1924 en París. El siglo XX fue un «siglo de extremos», ${ }^{18}$ en el que las exclusiones y boicots de los Juegos Olímpicos pusieron a prueba las declaraciones pacifistas del Movimiento Olímpico. ${ }^{19}$ A pesar de esto, la retórica idealista de la paz internacional y la armonía global inspiró la filosofía que apoyaba a la organización no sólo de los Juegos Olímpicos, sino también de los principales eventos deportivos internacionales, como la Copa de Fútbol. ${ }^{20}$ En la década de 1990, el Comité Olímpico Internacional revivió la antigua idea de la Tregua Olímpica en colaboración con las Naciones Unidas. En el año 2000 fundó la Fundación Internacional de la Tregua Olímpica que fijó como objetivo la promoción de la prevención y resolución de conflictos a través de los ideales deportivos y olímpicos, desplegando programas educativos y estrategias de comunicación para tal fin. ${ }^{21}$ Por lo tanto, la tregua olímpica no sólo tiene una dimensión política, que garantiza la pax olympica, sino también una importante dimensión educativa, aspirando a actuar como una «escuela para la paz». ${ }^{22}$

\section{La «escuela por la paz» olímpica}

La educación sobre la paz como término y campo independiente es relativamente reciente. Después de la Segunda Guerra Mundial, así como durante el conflicto posterior a la guerra fría en los Balcanes, la comunidad internacional incorporó la educación en las acciones de consolidación de la paz. Se invirtieron grandes cantidades, tanto en la reconstrucción de la infraestructura física (por ejemplo, escuelas destruidas) como en la llamada education for peace o peace education ${ }^{23}$ (educación

\footnotetext{
${ }^{16}$ Las citas pertenecen a Coubertin. Karl Lennartz ha analizado exhaustivamente la historia de los anillos olímpicos buscando si en la mente de Coubertin simbolizaban los cinco continentes. En desacuerdo en este sentido con otros investigadores: véase Karl Lennartz, «La historia de los anillos», Journal of Olympic History 10 (Dec. 2001 - Jan. 2002), pp. 29-61. También trabajo editado en versión en español como: Karl Lennartz, (2008) «La historia de los anillos», Citius, Altius, Fortius, Humanismo, Sociedad y Deporte. Investigaciones y Ensayos. Vol. 1-2008, pp.97-132.

${ }^{17}$ En Amberes (1920) cada paloma tenía una cinta con los colores de una de las naciones que participaban en los Juegos.

${ }^{18}$ Eric Hobsbawm, Age of Extremes. The Short Twentieth Century 1914-1991, London: Michael Joseph, 1994.

${ }^{19}$ M. Golden, «War and peace in the ancient and modern Olympics», Greece \& Rome, Vol. 58, No. 1, pp.1-13.

${ }^{20}$ A. Tomlinson-Chr. Young (eds.), National Identity and Global Sports Events. Culture, Politics, and Spectacle in the Olympics and the Football World Cup, State University of New York Press, 2006.

${ }^{21}$ Hay un gran debate sobre el significado y la práctica de la antigua tregua olímpica. Véase indicativamente Ramón Spaaij, «Olympic rings of peace? The Olympic movement, peacemaking and intercultural understanding» in Ramón Spaaij and Cindy Burleson (eds.), The Olympic movement and the Sport of Peacemaking, London-New York: Routledge, 2013,1 -14. 22 Jim Parry, «The Religio Athletae, Olympism and Peace», in K. Georgiadis-Ang. Syrigos (eds.), Olympic Truce. Sport as a Platform for Peace, Athens: International Olympic Truce Centre, 2009, pp. 45-47.

${ }^{23}$ A pesar de que existía educación para la paz a través de los siglos, su cultivo sistemático y su terminología de acompañamiento se desarrolló en la década de 1950, en combinación con la investigación científica sobre la paz en el contexto de la llamada «Peace Studies» (Harris, I., (2008). 'History of peace Education', in Encyclopedia of Peace Education, Teachers College, Columbia University, http://www.tc.ed/centers/epe (accessed 12.08.2015).
} 
para la paz). En cualquier caso, la construcción de la paz no se debe limitar a la reconstrucción social y política después de un acuerdo de paz, que formalmente marca el final de una guerra, ni a aplicarse en sociedades que han experimentado o están experimentando conflictos, sino en todos los sistemas educativos en combinación con la educación para la ciudadanía. Esta posición figura claramente en el artículo 26 de la Declaración Universal de Derechos Humanos, que fue adoptada por la Asamblea General de las Naciones Unidas en 1948:

«La educación se dirigirá al pleno desarrollo de la personalidad humana y al fortalecimiento del respeto de los derechos humanos y las libertades fundamentales. Promoverá la comprensión, la tolerancia y la amistad entre todas las naciones, grupos raciales o religiosos, y promoverá las actividades de Naciones Unidas para el mantenimiento de la paz» ${ }^{24}$.

El Movimiento Olímpico ha incorporado estos valores en cuanto al papel que debe desempeñar la educación a través del deporte y concede importancia a la función educativa del deporte. En este sentido, la Carta Olímpica (2018) establece que «el objetivo del Movimiento Olímpico es contribuir a la construcción de un mundo pacífico y mejor mediante la educación de los jóvenes a través del deporte practicado de acuerdo con el olimpismo y sus valores» ${ }^{25}$.

No se trata de una idea nueva, aunque expresa los valores del mundo moderno. Un elemento importante de los internacionalismos idealistas -que se puede encontrar en el Olimpismo- es la dimensión de la reforma educativa. Inspirándose en el optimismo educativo de la Ilustración, el internacionalismo a principios del siglo XIX aspiraba a cambiar el mundo a través de la educación. Los Juegos Olímpicos revividos fueron vistos como una «escuela para la paz», como un medio para la realización de valores e ideales morales. Además, el Olimpismo adoptó la forma de la Educación Olímpica, basándose en «cultivar el esfuerzo y cultivar la euritmia»y, en consecuencia, en el amor al exceso combinado con el amor por la moderación. ${ }^{26}$ La educación para la paz formaba parte de una ideología liberal mayor que determinaba los valores que deberían regir la educación en todo el mundo, principalmente, por supuesto, el mundo occidental, y que se condensaron como igualdad, equidad, integridad, racionalismo, respeto, libertad y comprensión internacional. ${ }^{27}$

Los valores olímpicos evolucionaron en el siglo XX, para corresponder al modelo de ciudadano de una democracia moderna, sentando las bases de la educación olímpica. Estos valores se difunden a través de un conjunto de eventos e instituciones olímpicas, con el Comité Olímpico Internacional como su orquestador clave. Sin embargo, es importante comprender que estos valores no conforman una doctrina religiosa, a pesar de que el olimpismo utilizó la terminología de la religión, y, por lo tanto, no permanecen firmes y sin cambios. Por el contrario, siguen las transformaciones ideológicas, sociales y políticas, cambiando a través del tiempo; tienen historicidad y no se perciben ni se aplican uniformemente en todo el mundo. El olimpismo actual no constituye un sistema filosófico cohesionado, sino más bien una «red de ideas, abierta a la interpretación y a las aplicaciones en diversos contextos ${ }^{28}$. Esta característica es crucial para la educación olímpica, cuya eficacia depende en gran medida de su adaptabilidad.

\footnotetext{
${ }^{24}$ UN 1948.

${ }^{25}$ Olympic Charter. In force as of 9 October 2018, IOC, 15.

${ }^{26}$ Lettres Olympiques V, Gazette de Lausanne, 325 (28 November 1918), pp. 1-2, in Müller (Ed.), Pierre de Coubertin, vol. II, op.cit., p. 15.

${ }^{27}$ Hoberman «Hacia una teoría del internacionalismo olímpico», Op.cit.

${ }^{28}$ Ramón Spaaij, «Olympic rings of peace? The Olympic movement, peacemaking and intercultural understanding», op.cit., p. 5.
}

Citius, Altius, Fortius Volumen 12 ne 2. Noviembre, 2019. I§SN. 2340-9886.

http://doi.org/10.15366/eitius2019.12.2 
En el mundo moderno, la educación para la paz «sobre el tema del multiculturalismo y la conciencia del sufrimiento de varios grupos involucrados en el conflicto, para promover la empatía por el sufrimiento de los demás y reducir las hostilidades ${ }^{29}$, recoge elementos que definitivamente no existían en las fases anteriores del Movimiento Olímpico. La educación para la paz está ahora vinculada con los derechos humanos, la comprensión intercultural, la resolución de conflictos, el fomento de la mentalidad y los comportamientos que permiten la coexistencia pacífica de grupos con una religión diferente, identidad nacional, capacidad física o discapacidad. El olimpismo conserva sus objetivos intervencionistas, su deseo reformista de «cambiar el mundo»; pero el mundo que quiere cambiar hoy es muy diferente del que quería cambiar hace un siglo y medio. Hoy en día, la utilización del deporte para combatir la discriminación social, el racismo y la xenofobia y promover la tolerancia hacia la diversidad y la reconciliación con la otredad comprenden intereses clave en la educación para la paz. Las iniciativas de la sociedad civil, las ONGs, las asociaciones de atletas, los movimientos sociales han añadido el deporte a su agenda, como uno de los medios para la educación mundial para la paz, como una de las herramientas para construir una cultura para la paz más allá de las fronteras estatales. ${ }^{30}$ Hay muchas razones. Primero, la competición deportiva en campos deportivos y estadios a menudo supone pretextos para la violencia racista y brotes de odio nacionalista en las gradas, pero también en el campo deportivo. En segundo lugar, el encuentro de atletas de todo el mundo en eventos deportivos internacionales ofrece una oportunidad única para superar prejuicios de género, nacionales y racistas, así como para promover un ejemplo único de reconciliación y noble competencia, carente de fanatismo. En tercer lugar, la inclusión del deporte en la educación para la paz podría tener mejores resultados que la educación teórica, si tenemos en cuenta la naturaleza lúdica del deporte y su popularidad entre los niños, incluso desde una edad preescolar. En cuarto lugar, muchos atletas masculinos y femeninos representan modelos a seguir para la juventud, de hecho, ciertos atletas son honrados como héroes nacionales, especialmente medallistas olímpicos; por lo tanto, se pueden utilizar para promulgar mentalidades que contrarresten el racismo y promuevan la paz.

Las investigaciones llevadas a cabo en muchos países europeos sobre la difusión de los valores olímpicos en la escuela no han dado lugar a resultados alentadores. Por ejemplo, en una investigación realizada en 2002 en cinco estados miembros de la UE, entre el 30 y el $40 \%$ de los jóvenes de ambos sexos opinaban que «si su oponente no juega limpio, es aceptable pagarle de la misma manera» ${ }^{31}$. En los resultados de una investigación realizada en Polonia en 2006, el 25\% de los jóvenes de 16 años no estaba de acuerdo con que los Juegos Olímpicos promocionaran la cultura de otras naciones, mientras que el 70-75\% estaba de acuerdo en que la motivación para participar en los Juegos Olímpicos era el honor de representar a un país. ${ }^{32}$ Desde el renacimiento de los Juegos Olímpicos, el equilibrio entre nacionalismo e internacionalismo ha sido frágil y solo en raras ocasiones el estadio logró convertirse en un espacio simbólico para la paz. De hecho, muchos investigadores han subrayado la falta de eficacia con respecto a la implementación de la Tregua Olímpica. Como observa Michael Llewellyn Smith, la tregua «es un concepto agradable que no sobreviviría a las duras realidades de las relaciones interestatales modernas ${ }^{33}$. A pesar de lo cual, o quizás, precisamente por este hecho, la educación para la paz constituye una parte necesaria de la educación olímpica.

\footnotetext{
${ }^{29}$ Harris, Op.cit. Pp. 3-4.

${ }^{30}$ Para ver ejemplos, véase Kamperidou, Eirini. Koinoniko fylo, koinoniko kefalaio, polypolitismikotita kai athlitismos [Social gender, social capital, multicultarilism and sports, Athens: Telethrion, 2012.

${ }^{31}$ Los países participantes en la investigación fueron la República Checa, Finlandia, Alemania, Esthonia y Hungría. Bronikowski-Bronikowska, «Can Olympic education...» in K. Georgiadis-Ang. Syrigos (eds.), Olympic Truce. Sport as a Platform for Peace, Athens: International Olympic Truce Centre, 2009, p.98.

${ }^{32}$ Ibid., p.99.

${ }^{33}$ M. Golden, «War and peace in the ancient and modern Olympics», op.cit., p.7.
} 
En una sociedad globalizada, en la que la competencia por la distinción a cualquier costo se está haciendo más fuerte, la educación, no sólo la educación olímpica, debe desarrollar un espíritu de colaboración y espíritu de equipo en los niños desde una edad temprana. Antes de empezar a competir, los niños deben aprender a trabajar juntos y a respetarse mutuamente. El aula es un laboratorio para el desarrollo moral y la formación de la personalidad, donde el aprendizaje tiene un significado que se puede transferir a la vida social fuera de la escuela. El papel del educador es crucial en este procedimiento, porque no es suficiente ofrecer a los alumnos un aprendizaje teórico, sino que debe involucrarlos emocional e intelectualmente en el aprendizaje a través de la empatía. La manera en que se comportan los educadores tiene un peso igual o incluso mayor que lo que dicen cuando enseñan a los niños.

Obviamente, la educación para la paz no es el tema de una clase, sino más bien un tema educativo común que se ejecuta a través de todas las materias del plan de estudios. No es realista perseguir el desarrollo de los valores morales y las mentalidades en los niños enseñándoles sólo en una materia. La educación olímpica, por lo tanto, debe trabajar en conjunto y comunicarse con otras materias, invirtiendo más en el aspecto práctico que en el aspecto teórico del aprendizaje. Es más probable que los niños aprendan a comportarse a través de los deportes, en lugar de los libros, y que los apliquen fuera de la escuela.

Hay una cuestión seria que debe abordarse cuando implementamos un programa de educación olímpica con aspiraciones globales. ¿Cómo aplicamos un sistema de valores morales y modelos culturales comunes - del olimpismo en el caso que nos ocupa- en todos los países y todas las naciones del mundo? El conjunto de reglas según las cuales se desarrolla un evento deportivo es distinto al hecho de que estas reglas sean consideraciones morales, y sirvan para que, sobre su base, las personas actúen en su vida social, fuera del estadio y el campo deportivo. Sin embargo, la paz, con todos los factores que conlleva y más allá de las diferencias culturales, es el único valor olímpico que puede considerarse verdaderamente global y que, a través del Movimiento Olímpico, puede aplicarse en cualquier rincón del planeta. Los educadores pueden ser quienes estén llamados a tener un papel fundamental como «embajadores de la paz», dado su protagonismo en el desarrollo de las próximas generaciones. Su posición y labor pueden favorecer que estas sean capaces de vivir en armonía, construyendo un futuro donde la paz sea más probable que la guerra, gran utopía de nuestra sociedad.

\section{Bibliografía}

- Tomlinson-Chr. Young (eds.) (2006), National Identity and Global Sports Events. Culture, Politics, and Spectacle in the Olympics and the Football World Cup, State University of New York Press.

- Bronikowski-Bronikowska, (2009): "Can Olympic education...” in K. Georgiadis-Ang. Syrigos (eds.), Olympic Truce. Sport as a Platform for Peace, Athens: International Olympic Truce Centre.

— Clastres, P., Dietschy, P.et Laget, S. (2004): La France et l'Olympisme, Paris : ADPF, 2004.

- Cooper, Sandi E. (1991): Patriotic Pacifism. Waging War on War in Europe 1815-1914, New York-Oxford: Oxford University Press. 
- Coubertin (de), P. (1918). Lettres Olympiques V, Gazette de Lausanne, 325 (28 November 1918), pp. 1-2, in Müller (ed.), Pierre de Coubertin, vol. II, op.cit., p. 15.

- Coubertin (de), P. (1901). Notes sur l'éducation publique. En N. Müller (Ed.) Pierre de Coubertin 1863-1937 - Olympism: Selected Writings. Lausanne: International Olympic Committee, 2000, note 41 .

- Golden, M. (2011). War and peace in the ancient and modern Olympics. Greece \& Rome, Vol. 58, No. 1, pp.1-13. https://doi.org/10.1017/S0017383510000495

- Harris, I., (2008). 'History of peace Education', in Encyclopedia of Peace Education, Teachers College, Columbia University, http://www.tc.ed/centers/epe (accessed 12.08.2015).

- Hoberman, John (1995) "Toward a Theory of Olympic Internationalism", Journal of Sport History 22/1.

- Hobsbawm, Eric (1994): Age of Extremes. The Short Twentieth Century 1914-1991, London: Michael Joseph.

- Kamperidou, Eirini. (2012) Koinoniko fylo, koinoniko kefalaio, polypolitismikotita kai athlitismos [Social gender, social capital, multicultarilism and sports, Athens: Telethrion.

- Koulouri, Christina (2004) "Introduction: Rewriting the History of the Olympic Games" in idem (ed.), Athens, Olympic City, 1896-1906, Athens: International Olympic Academy.

— Lennartz, Karl, (2008) "La historia de los anillos", Citius, Altius, Fortius, Humanismo, Sociedad y Deporte. Investigaciones y Ensayos. Vol. 1-2008, pp.97-132.

- Lennartz, Karl,(2002) “The Story of the Rings”,Journal of Olympic History 10 (Dec. 2001 - Jan. 2002), pp. 29-61.

- MacAloon, John (1996) "Olympic Ceremonies as a Setting for Intercultural Exchange" in M. De Moragas -J. MacAloon - M. Llinés (eds.), Olympic Ceremonies. Historical Continuity and Cultural Exchange, Lausanne: IOC.

- Müller ,N. (ed.) (2000), Pierre de Coubertin 1863-1937.Olympism: Selected Writings. Lausanne: International Olympic Committee.

- Olympic Charter. In force as of 9 October 2018, IOC, 15.

- Parry, Jim (2009) "The Religio Athletae, Olympism and Peace”, in K. Georgiadis-Ang. Syrigos (eds.), Olympic Truce. Sport as a Platform for Peace, Athens: International Olympic Truce Centre, pp. 45-47.

- Quanz, Dietrich R. (1993) "Civic Pacifism and Sports-Based Internationalism: Framework for the Founding of the International Olympic Committee", Olympika: The International Journal of Olympic Studies 2 (1993). 
- Rioux, G., (ed.), Pierre de Coubertin. Textes choisis, vol. 1: Révélation, Weidmann, ZurichHildersheim-New York.

- Spaaij, Ramón (2013): "Olympic rings of peace? The Olympic movement, peacemaking and intercultural understanding" in Spaaij, Ramón and Burleson, Cindy (eds.), The Olympic movement and the Sport of Peacemaking, London-New York: Routledge, pp. 1-14. 